\title{
Revisiting CO2 Emissions Convergence in G18 Countries
}

\author{
Jiangpeng Lin \\ School of Finance, Hubei University of China, Wuhan, CHINA \\ Roula Inglesl-Lotz* \\ Department of Economics, University of Pretoria, Pretoria, South Africa \\ Tsangyao Chang \\ Department of Finance, Feng Chia University, Taichung, TAIWAN
}

\begin{abstract}
This study revisits whether $\mathrm{CO}_{2}$ emissions converge in G18 countries over the period of 1950-2013.

To work on this empirical analysis, we employ a more powerful Quantile unit root test with per capita $\mathrm{CO}_{2}$ emissions. While conventional unit root tests fail to reject convergence in $\mathrm{CO}_{2}$ emissions in these G18 countries, Quantile unit root test results demonstrate $\mathrm{CO}_{2}$ emissions converged in 5 of these G18 countries (i.e., Australia, Brazil, Canada, Germany and India). Our empirical results have important policy implications for the governments of G18 countries to direct efficient and effective energy policies to reduce the $\mathrm{CO}_{2}$ emissions.
\end{abstract}

Keywords: Per Capita CO2 Emissions; Per Capita Emissions; G18 Countries; Convergence; Quantile Unit Root Test

\section{Introduction}

In order to fight the continuously increasing greenhouse gases emissions, G20 countries seem to be in close collaboration through UNFCC and COP discussions on how to combat the global warming

** Corresponding author: Email: roula.inglesi-lotz@up.ac.za 
phenomenon in search of optimal and effective energy and environmental strategies. Understanding fully the time series properties and evolution of greenhouse gas emissions and particularly of carbon dioxide $\mathrm{CO} 2$ and their geographic distribution will be of high importance for the international climate change negotiations and the global political economy (El-Montasser et al, 2015). The paper offers answers to that direction by examining whether the $\mathrm{CO}_{2}$ emissions converge and for which countries. If $\mathrm{CO}_{2}$ emissions converged, then the policymakers can continue with the same approaches and policy implementations as currently. Otherwise, it means that the policymakers must reduce carbon dioxide emission rigidly according to the difference of emission amount. Policy proposals in these economies must reexamine the existing reducing policies and put forward some reasonable suggestions to obtain sustainable development (Sun et al., 2016).

To investigate the magnitude of emissions convergence empirically, recently researchers have relied on conventional unit root tests (i.e., ADF, PP and KPSS tests) to assess if shocks to $\mathrm{CO}_{2}$ emissions are permanent, a feature to be evident against convergence. However, previous studies usually focus on the average converging behavior of $\mathrm{CO}_{2}$ emissions without considering the influence of various sizes of shocks on $\mathrm{CO}_{2}$ emissions. In other words, the speed of convergence in $\mathrm{CO}_{2}$ emissions is usually assumed to be constant, irrespective of the size and direction of the shock. As a result, the commonly used conventional unit root tests (i.e., ADF, PP and KPSS) possibly lead to a widespread failure in the rejection of unit-root null hypothesis of convergence. This paper intends to deal with this kind of deficiency by employing a more powerful Quantile Unit Root test to 
enhance its estimation and testing accuracy.

This study contributes to this line of research (Aldy, 2006a; Aldy, 2006b; Barassi et al, 2008;

Barassi et al, 2011; Camarero et al, 2011; Chang and Lee, 2008; Huang and Meng, 2013; Jobert et al., 2010; Lee and Chang, 2009; Li et al, 2014; Li and Lin, 2013; McKibbin and Stegman, 2005; Ordas-Criado and Grether, 2011; Panopoulou and Pantelidis, 2009; Romero-Avila, 2008; Strazicich and List, 2003; Sun et al 2016; Wang and Zhang, 2014; Westerlund and Basher, 2007; Yavuz and Yilanci, 2013; Zhao et al, 2015) by determining whether $\mathrm{CO}_{2}$ emissions convergence in G18 countries by using a more powerful Quanitle Unit Root test as proposed by Koenker and Xiao (2004).

The studies however concerned with emissions convergence could not reach a consensus on its existence. Aldy (2006a) explained, for instance, that no convergence can be concluded when country and time period is extended. Also, Lee and Chang (2008) only some of the OECD countries could confirm convergence while Barassi et al. (2008) concluded that emissions convergence could not be achieved in OECD countries at all. The issue of the speed of convergence is also discussed in the literature with Barassi et al. (2011) showing that the rate is quite slow for 13 out of 18 OECD countries.

\section{Methodology}

\subsection{Convergence Tests}

The methodology is rooted in the work of Evans (1998), who introduces a particular notion of 
convergence, which implies that the long-run $\mathrm{CO}_{2}$ gap between any two regions must be stationary. To formalize the idea empirically, suppose that $y_{i t}$ the $\log$ of $\mathrm{CO}_{2}$ emissions for state $i=G 18$ countries at time $t=1, \ldots, T$, is non-stationary, and thus exhibits a unit root. Then a pair-wise convergence is said to occur if, for any pair of states $i$ and $j$, the difference, $y_{i t}-y_{j t}$, is stationary so that $y_{i t}$ and $y_{j t}$ are cointegrated. Specifically, this notion of pair-wise convergence is equivalent to the condition that the difference between the individual series and their mean value at each point in time is stationary.

This hypothesis could be tested using the following regression

$$
y_{i t}=\alpha_{i}+\tau_{i} t+\varphi_{i} y_{i t-1}+e_{i t}
$$

where $\oint_{i t}=y_{i t}-\frac{1}{N} \sum_{j=1}^{N} y_{j t}$ (relative per capita $\mathrm{CO}_{2}$ emissions), $\alpha_{i}$ and $\tau_{i}$ are state specific intercept and trend terms, and $e_{i t}$ is a disturbance term that may by correlated across both $i$ and $t$. The key parameter is $\varphi_{i}$ which measures the degree of the convergence. If $\varphi_{i}=1$ then state $i$ has a unit root and is thus non-convergent, whereas, if $\varphi_{i}<1$ then state $i$ is convergent. The exact hypothesis to be tested is given as follows.

$$
H_{0}: \varphi_{i}=1 \text { for all } i \text { versus } H_{1}: \varphi_{i}<1 \text { for some } i \text {. }
$$

A rejection of the null should therefore be taken as evidence in favor of convergence for at least one state, whereas a non-rejection should be taken as evidence of non-convergence for the whole panel.

\subsection{Quantile Unit Root Test}

In line with Koenker and Xiao (2004) and let $r p c 2_{t}$ denote the relative per capita $\mathrm{CO}_{2}$ emissions 
among G18 countries and $\varepsilon_{t}$ a serially uncorrelated error term. An AR(q) process with a drift is given by:

$r p c 2_{t}=a+\sum_{i=1}^{q} \gamma_{i} r p c 2_{t-1}+\varepsilon_{t}, \quad t=q+1, q+2, \ldots, n$.

We can rewrite Equation (3) as follows:

$r p c 2_{t}=\alpha r p c 2_{t-1}+a+\sum_{i=1}^{q-1} \phi_{i} \Delta r p c 2_{t-i}+\varepsilon_{t}$

Here we can run the usual unit root test. If $\alpha=1$ then the real interest rate differential has a unit root and, therefore, shocks have permanent effects on relative per capita $\mathrm{CO}_{2}$ emissions. If we have $\alpha<1$, then relative per capita $\mathrm{CO}_{2}$ emissions are stationary. In this case shocks have only temporary effects on relative per capita $\mathrm{CO}_{2}$ emissions. This means that $\mathrm{CO}_{2}$ emissions convergence holds true under this situation.

To gain more detailed estimates to analyze persistence, the focus cannot be only on the conditional mean but also in the tails of the conditional distribution of $r p c 2_{t}$ and here equation (4) can be estimated using quantile autoregression methods. The $\tau-$ th conditional quantile is defined as the value $Q_{\tau}\left(r p c 2_{t} \mid r p c 2_{t-1}, \ldots, r p c 2_{t-q}\right)$ such that the probability of relative per capita $\mathrm{CO} 2$ emissions conditional on its recent and past history will be less than $Q_{\tau}\left(r p c 2_{t} \mid r p c 2_{t-1}, \ldots, r p c 2_{t-q}\right)$ is $\tau$. For example, if relative per capita $\mathrm{CO}_{2}$ emissions are at very high (low) relative to recent relative per capita $\mathrm{CO}_{2}$ emissions this means that a large positive (negative) shock has occurred and that rid ${ }_{t}$ is located above (below) the mean conditional on past observations $r p c 2_{t-1}, \ldots, r p c 2_{t-q}$ somewhere in the upper (lower) conditional quantiles. 
The $\mathrm{AR}(\mathrm{q})$ process of relative per capita $\mathrm{CO} 2$ emissions at quantile $\tau$ can be written as:

$Q_{\tau}\left(r p c 2_{t} \mid r p c 2_{t-1}, \ldots, r p c 2_{t-q}\right)=\alpha(\tau) r p c 2_{t-1}+a(\tau)+\sum_{i=1}^{q-1} \phi_{i}(\tau) \Delta r p c 2_{t-i}$

By estimating Equation (5) at different quantiles $\tau \in(0,1)$ we can get a set of estimates of the persistence measure as $\alpha(\tau)$. We can test $\alpha(\tau)=1$ at different values of $\tau$ to analyze the persistence of the relative per capita $\mathrm{CO}_{2}$ emissions impact of positive and negative shocks and shocks of different magnitude using the quantile autoregression based unit root test proposed by Koenker and Xiao (2004).

Let $\alpha(\tau)$ be the quantile regression estimator. To test $H_{0}: \alpha(\tau)=1$ we use the $\mathrm{t}$-stat for $\alpha(\tau)$ proposed by Koenker and Xiao (2004) which can be written as $t_{n}(\tau)=\frac{f\left(F^{-1}(\tau)\right)}{\sqrt{\tau(1-\tau)}}\left(r p c 2_{-1}^{\prime} M_{Z} r p c 2_{-1}\right)^{1 / 2}(\alpha(\tau)-1)$

where $f(u)$ and $F(u)$ are the probability and cumulative density functions of $\varepsilon_{t}, r p c 2_{-1}$ is the vector of lagged relative per capita $\mathrm{CO}_{2}$ emissions and $\mathrm{MZ}$ is the projection matrix onto the space orthogonal to $Z=\left(1, \Delta r p c 2_{t-1}, \Delta r p c 2_{t-2}, \ldots, \Delta r p c 2_{t-q+1}\right)$. We use the results derived by Koenker and Xiao (2004) to find the critical values of $t_{n}(\tau)$ for different quantile levels. We can estimate $f\left(F^{-1}(\tau)\right)$ following the rule given in Koenker and Xiao (2004). Besides, by allowing for asymmetric effects of shocks on real interest rate differential and one of an important advantage of Quantile unit root tests over standard unit root tests is that it has more power (Koenker and Xiao, 2004).

\section{Data}

The per capita $\mathrm{CO} 2$ emissions series is derived from the CDIAC (http://cdiac.ornl.gov/CO2 
Emission/times/national - Carbon Dioxide Information Analysis Center) with the data ending in 2013 for G18 countries (i.e., Argentina, Australia, Brazil, Canada, China, France, Germany, India, Indonesia, Italy, Japan, Mexico, Saudi Arabia, South Africa, South Korea, Turkey, the UK and the US). Due to data availability for G18 countries, that we do not include Russia and the European Union and we start our sample only in 1950. According to United States Environmental Protection Agency reports (EPA, 2017) that CO2 emission from these G18 countries represented roughly about $84 \%$ of total world CO2 emissions in 2014.

The necessity of using per capita measures in the analysis of $\mathrm{CO} 2$ emissions has been emphasized by Aldy (2006a) because per capita basis is a direct measure of human welfare. Also, as mentioned by McKibbin and Stegman (2005), individual activities such as car use cause greenhouse gases, so it can be assured that a per capita measure is necessary.

Figure 1 demonstrates time series plots of per capita $\mathrm{CO}_{2}$ emissions in these 18 OECD countries further confirm our initial findings. Jarque-Bera statistics indicate that $\mathrm{CO}_{2}$ emissions are non-normal for most the OECD countries with the exception of Argentina, Brazil, Germany, South Africa, Turkey, the UK and the United States. As pointed by Koenker and Xiao (2004), the QAR-based unit root test has higher power than conventional unit root tests, because the QAR-based unit root test is superior to standard unit root tests in case of departure from Gaussian residuals and these further confirm the use of our Qunatile Unit Root test 


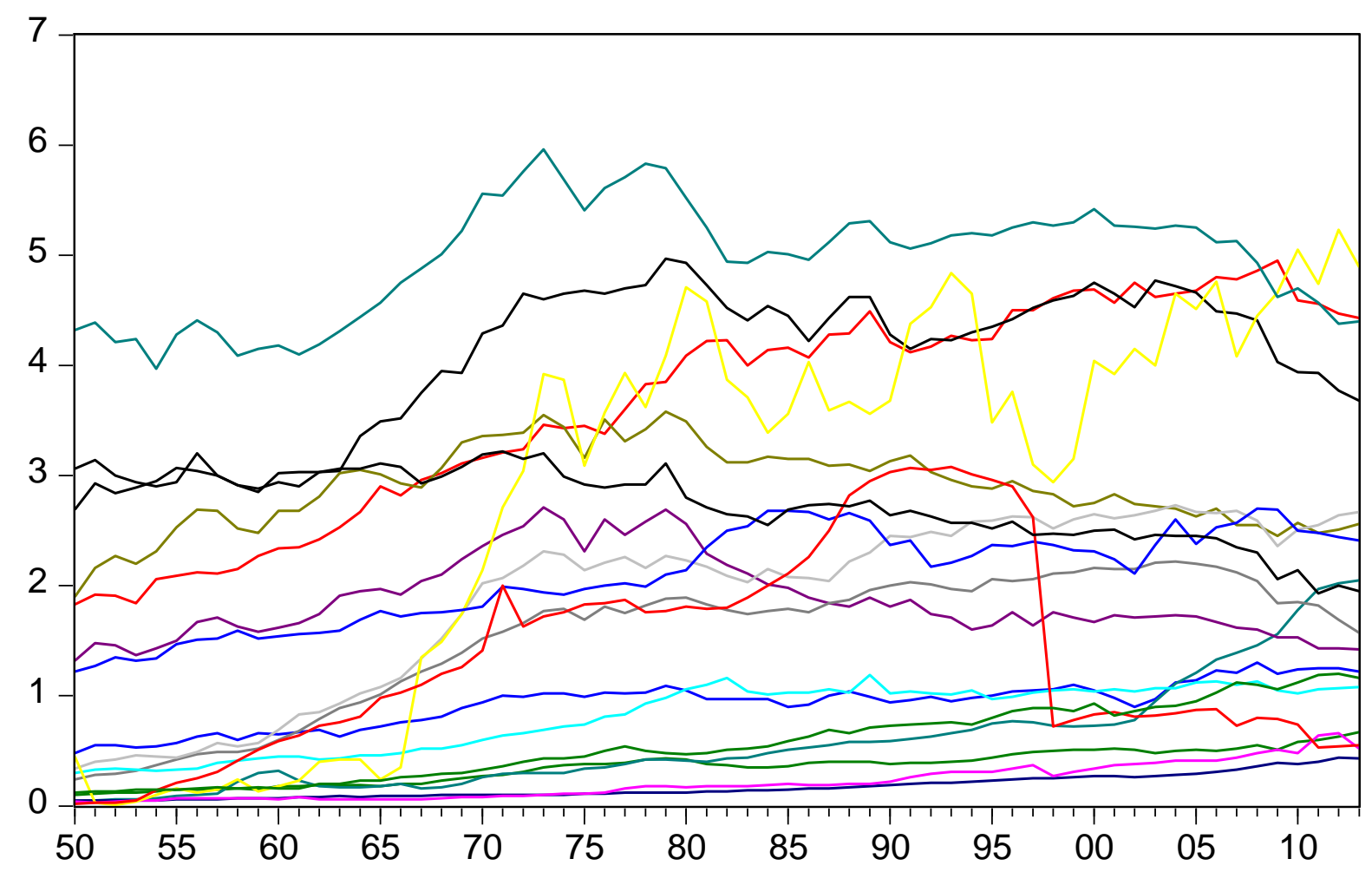

Figure 1. Plots of Per Capita CO2 Emissions in G18 Countries

\begin{tabular}{|lll|}
\hline ARGENTINA & AUSTRALIA & - BRAZIL \\
CANADA & CHINA & FRANCE \\
GERMANY & INDIA & INDNESIA \\
GEXICO \\
ITALY & JAPAN & SOUTH_KOREA \\
SAUDI_ARABIA & SOUTH_AFRICA & US \\
TURKEY & UK & - US \\
\hline
\end{tabular}

\section{Empirical Results and Policy Implications}

\subsection{Traditional Unit Root Tests}

Several univariate time series unit root tests - the ADF test (Dickey and Fuller, 1980), PP test (Phillips and Perron, 1989) and KPSS tests (Kwiatkowski et al., 1992) - are first employed to examine the null of a unit root for relative per $\mathrm{CO}_{2}$ emissions for $\mathrm{G} 18$ countries. The results of the three univariate unit root tests lead us to conclude that relative per $\mathrm{CO}_{2}$ emissions of the G18 countries contain unit roots (see Table 1). Besides allowing for asymmetric effects of shocks on 
Table 1. Univariate unit root tests

\begin{tabular}{|c|c|c|c|c|c|c|}
\hline & \multicolumn{3}{|c|}{ Level } & \multicolumn{3}{|c|}{ 1st difference } \\
\hline & ADF & PP & KPSS & ADF & PP & KPSS \\
\hline ARGENTINA & $-1.629111(0)$ & $-1.629111(0)$ & $0.641509(6)^{* *}$ & $-6.554042(0)^{* * *}$ & $-6.524974(2)^{* * *}$ & $0.537266(0)^{* *}$ \\
\hline AUSTRALIA & $-1.363172(0)$ & $-1.363172(0)$ & $0.975566(6)^{* * *}$ & $-9.194711(0)^{* * *}$ & $-9.196581(1)^{* * *}$ & $0.096334(0)$ \\
\hline BRAZIL & $-2.139295(0)$ & $-2.000321(4)$ & $0.649840(6)^{* *}$ & $-5.932199(0)^{* * *}$ & $-6.149241(4)^{* * *}$ & $0.535036(5)^{* *}$ \\
\hline CANADA & $-1.102500(0)$ & $-1.245095(3)$ & $0.232052(6)$ & $-7.772043(0)^{* * *}$ & $-7.773425(2)^{* * *}$ & $0.276083(2)$ \\
\hline CHINA & $0.591141(1)$ & $0.774677(5)$ & $0.281560(6)$ & $-4.252460(0)^{* * *}$ & $-4.228451(3)^{* * *}$ & $0.735247(5)^{* *}$ \\
\hline FRANCE & $-0.067054(0)$ & $0.082592(2)$ & $0.894570(6)^{* * *}$ & $-9.208640(0)^{* * *}$ & $-9.208553(2)^{* * *}$ & $0.260518(1)$ \\
\hline GERMANY & $-0.564788(0)$ & $-0.416651(3)$ & $0.856354(6)^{* * *}$ & $-8.441531(0)^{* * *}$ & $-8.904163(6)^{* * *}$ & $0.459427(4)^{*}$ \\
\hline INDIA & $-2.355669(0)$ & $-2.118078(4)$ & $0.645468(6)^{* *}$ & $-5.914320(0)^{* * *}$ & $-6.105537(4)^{* * *}$ & $0.595564(4)^{* *}$ \\
\hline INDONESIA & $-2.161717(0)$ & $-2.037323(4)$ & $0.543986(6)^{* *}$ & $-6.600151(0)^{* * *}$ & $-6.834196(4)^{* * *}$ & $0.577044(4)^{* *}$ \\
\hline ITALY & $-1.844605(0)$ & $-1.873278(4)$ & $0.752894(6)^{* * *}$ & $-7.121036(0)^{* * *}$ & $-7.301738(4)^{* * *}$ & $0.489828(4)^{* *}$ \\
\hline JAPAN & $-1.196273(0)$ & $-1.198564(1)$ & $0.895490(6)^{* * *}$ & $-7.507071(0)^{* * *}$ & $-7.507071(0)^{* * *}$ & $0.104651(1)$ \\
\hline MEXICO & $-2.041691(0)$ & $-2.054077(1)$ & $0.373446(6)^{*}$ & $-7.530078(0)^{* * *}$ & $-7.530207(1)^{* * *}$ & $0.244840(1)$ \\
\hline SAUDI_ARABIA & $-0.991148(0)$ & $-0.937146(3)$ & $0.846137(6)^{* * *}$ & $-8.241825(0)^{* * *}$ & $-8.290322(4)^{* * *}$ & $0.068804(0)$ \\
\hline
\end{tabular}


relative per $\mathrm{CO}_{2}$ emissions, as we know that one of an important advantage of Quantile unit root tests over standard unit root tests is that they have more power (Koenker and Xiao, 2004), and hence, be more helpful.

\subsection{Quantile Unit Root Tests and Policy Implications}

Due to the deficiency of conventional unit root tests, in the following we employ a newly developed Quantile unit root test proposed by Koenker and Xiao (2004) to enhance its estimation accuracy. To test the null of $\alpha(\tau)=1$ for $\tau=0.1,0.2,0.3,04, \ldots, 0.9$ more formally, we use the t-statistic $\left(t_{n}(\tau)\right)$ based on Eq. (5). Table 2 shows the point estimates, p-value, half-life of a shock, and $Q K S$ for each country. We find that $H_{0}: \alpha(\tau)=1$ can be rejected at the $10 \%$ significance level over the whole conditional $r p c 2_{t}$ distribution based on Quantile Kolmogorov-Smirnov test $(Q K S)$ for 5 out of 18 countries (i.e., Australia, Brazil, Canada, Germany and India). The test result confirms that all types of shocks to $r p c 2_{t}$ lead to only temporary effects and $\mathrm{CO}_{2}$ emissions convergence did hold true in these five countries. Empirical results lead us to the conclusion that relative per capita $\mathrm{CO}_{2}$ emissions did converge in some G18 countries (i.e., Australia, Brazil, Canada, Germany and India).

Table 3 also shows the persistent estimates of $\alpha(\tau)$ for $\tau=0.1,0.2,0.3, \ldots \ldots, 0.9$ in each country. The persistence parameter estimates are quite close to one for most of the quantiles considered in France, Saudi Arabia, South Korea and Turkey. The persistent point estimate is slightly above one at the higher tail quantile for Australia, France, Italy, South Korea. To take a closer look at the estimated half-life of a shock and we find that it increases from lower quantile to higher quantile. 
Table 2: Quantile Unit Root Test Results

\begin{tabular}{|c|c|c|c|c|c|c|c|c|c|}
\hline Quantile & 0.10 & 0.20 & 0.30 & 0.40 & 0.50 & 0.60 & 0.70 & 0.80 & 0.90 \\
\hline \multicolumn{10}{|c|}{ ARGENTINA } \\
\hline$\alpha(\tau)$ & 0.9661 & 0.9732 & 0.9771 & 0.9704 & 0.9215 & 0.9252 & 0.9309 & 0.9297 & 0.9389 \\
\hline t-stat. & -0.8274 & -0.5614 & -0.4711 & -0.6029 & -1.8852 & -1.8834 & -2.1075 & -1.7649 & -1.9117 \\
\hline C.V. & -2.1764 & -2.5266 & -2.5097 & -2.5269 & -2.7231 & -2.7050 & -2.7015 & -2.5657 & -2.1566 \\
\hline \multicolumn{10}{|l|}{$\mathrm{H}-\mathrm{L}$} \\
\hline QKS test & 2.1075 & & & & & & & & \\
\hline \multicolumn{10}{|c|}{ AUSTRALIA } \\
\hline$\alpha(\tau)$ & 0.8668 & 0.9433 & 0.9627 & 0.9989 & 1.0079 & 0.9976 & 1.0014 & 1.0313 & 0.9942 \\
\hline t-stat. & -2.8689 & -1.1761 & -1.0092 & -0.0363 & 0.2307 & -0.0592 & 0.0350 & 0.7690 & -0.1863 \\
\hline C.V. & -2.1223 & -2.3384 & -2.3177 & -2.3684 & -2.5553 & -2.4369 & -2.4363 & -2.4058 & -2.5978 \\
\hline $\mathrm{H}-\mathrm{L}$ & 4.8490 & & & & & & & & \\
\hline QKS test & 2.8689 & & & & & & & & \\
\hline \multicolumn{10}{|c|}{ BRAZIL } \\
\hline$\alpha(\tau)$ & 0.9135 & 0.9727 & 0.9644 & 0.9605 & 0.9395 & 0.9328 & 0.9264 & 0.8879 & 0.8933 \\
\hline t-stat. & 2.2238 & -0.9993 & -0.8917 & -0.9768 & -1.3118 & -1.5389 & -1.5555 & -3.2906 & 2.5070 \\
\hline C.V. & $\mathrm{NaN}$ & -2.5711 & -2.5295 & -2.5509 & -2.7354 & -2.6301 & -2.4354 & -2.2589 & -2.1200 \\
\hline $\mathrm{H}-\mathrm{L}$ & & & & & & & & 5.8299 & 6.1431 \\
\hline QKS test & 3.2906 & & & & & & & & \\
\hline \multicolumn{10}{|c|}{ CANADA } \\
\hline$\alpha(\tau)$ & 0.8521 & 0.8247 & 0.7136 & 0.7488 & 0.7320 & 0.8771 & 0.7665 & 0.8969 & 0.9712 \\
\hline t-stat. & 1.1848 & -1.9871 & -2.9618 & -2.3415 & -2.2833 & -0.9545 & -1.9287 & -1.0372 & 0.2423 \\
\hline C.V. & -2.1200 & -2.1666 & -2.5194 & -2.5998 & -2.5390 & -2.5201 & -2.4995 & -2.5494 & -2.2732 \\
\hline $\mathrm{H}-\mathrm{L}$ & & & 2.0542 & & & & & & \\
\hline QKS test & 2.9618 & & & & & & & & \\
\hline \multicolumn{10}{|c|}{ CHINA } \\
\hline$\alpha(\tau)$ & 0.9495 & 0.9510 & 0.9613 & 0.9620 & 0.9517 & 0.9755 & 0.9853 & 0.9675 & 0.9810 \\
\hline t-stat. & 1.3411 & -1.4842 & -0.8324 & -0.8558 & -1.0129 & -0.4543 & -0.3050 & -0.7372 & 0.4116 \\
\hline C.V. & -2.1623 & -2.4754 & -2.6013 & -2.6007 & -2.5590 & -2.6002 & -2.5378 & -2.6220 & -2.1619 \\
\hline \multicolumn{10}{|l|}{$\mathrm{H}-\mathrm{L}$} \\
\hline QKS test & 1.4842 & & & & & & & & \\
\hline \multicolumn{10}{|c|}{ FRANCE } \\
\hline$\alpha(\tau)$ & 0.9046 & 1.0049 & 0.9972 & 1.0270 & 1.0124 & 1.0205 & 1.0344 & 1.0302 & 1.0562 \\
\hline t-stat. & -2.5287 & 0.1359 & -0.0660 & 0.8226 & 0.4270 & 0.6685 & 0.9295 & 0.5788 & 1.1610 \\
\hline C.V. & -2.1200 & -2.5197 & -2.5745 & -2.6188 & -2.6965 & -2.5316 & -2.4366 & -2.4882 & -2.4015 \\
\hline \multicolumn{10}{|l|}{$\mathrm{H}-\mathrm{L}$} \\
\hline QKS test & 2.5287 & & & & & & & & \\
\hline \multicolumn{10}{|c|}{ GERMANY } \\
\hline$\alpha(\tau)$ & 0.8928 & 0.9900 & 1.0357 & 1.0210 & 1.0377 & 1.0649 & 1.0484 & 1.1044 & 1.0205 \\
\hline t-stat. & -3.0939 & -0.2199 & 0.7643 & 0.4152 & 0.6766 & 1.1679 & 0.7858 & 1.7156 & 0.8584 \\
\hline C.V. & -2.1200 & -2.4799 & -2.4019 & -2.3369 & -2.7263 & -2.3269 & -2.6242 & -2.5759 & -2.6298 \\
\hline $\mathrm{H}-\mathrm{L}$ & 6.1128 & & & & & & & & \\
\hline QKS test & 3.0939 & & & & & & & & \\
\hline
\end{tabular}




\begin{tabular}{|c|c|c|c|c|c|c|c|c|c|}
\hline Quantile & 0.10 & 0.20 & 0.30 & 0.40 & 0.50 & 0.60 & 0.70 & 0.80 & 0.90 \\
\hline \multicolumn{10}{|c|}{ INDIA } \\
\hline$\alpha(\tau)$ & 0.9170 & 0.9180 & 0.9701 & 0.9627 & 0.9395 & 0.9409 & 0.9102 & 0.9048 & 0.9084 \\
\hline t-stat. & 2.1674 & -3.8928 & -0.9980 & -1.1679 & -1.3144 & -1.3208 & -2.2636 & -2.8945 & 2.1813 \\
\hline C.V. & -2.1200 & -2.3407 & -2.5786 & -2.6704 & -2.6836 & $\mathrm{NaN}$ & -2.6345 & -2.4115 & -2.1200 \\
\hline $\mathrm{H}-\mathrm{L}$ & & 8.1015 & & & & & & 6.9286 & \\
\hline QKS test & 3.8928 & & & & & & & & \\
\hline \multicolumn{10}{|c|}{ INDONESIA } \\
\hline$\alpha(\tau)$ & 1.0219 & 0.9768 & 0.9614 & 0.9729 & 0.9546 & 0.9688 & 0.9461 & 0.9239 & 0.9256 \\
\hline t-stat. & 0.6591 & -0.6750 & -1.2855 & -0.8549 & -1.5424 & -0.9747 & -1.5801 & -2.0276 & -1.0474 \\
\hline C.V. & -2.3523 & -2.4066 & -2.3981 & -2.5919 & -2.6943 & -2.7439 & -2.6585 & -2.6246 & -2.4679 \\
\hline \multicolumn{10}{|l|}{$\mathrm{H}-\mathrm{L}$} \\
\hline QKS test & 2.0276 & & & & & & & & \\
\hline \multicolumn{10}{|c|}{ ITALY } \\
\hline$\alpha(\tau)$ & 0.9220 & 0.9130 & 0.8997 & 0.9225 & 0.9497 & 0.9587 & 0.9744 & 0.9825 & 1.0142 \\
\hline t-stat. & -1.6623 & -1.8234 & -2.2130 & -1.8912 & -1.2524 & -0.9026 & -0.4971 & -0.2785 & 0.2290 \\
\hline C.V. & -2.3510 & -2.1949 & -2.4582 & -2.2848 & -2.3489 & -2.5287 & -2.6111 & -2.5746 & -2.2989 \\
\hline \multicolumn{10}{|l|}{$\mathrm{H}-\mathrm{L}$} \\
\hline QKS test & 2.2130 & & & & & & & & \\
\hline \multicolumn{10}{|c|}{ JAPAN } \\
\hline$\alpha(\tau)$ & 0.9623 & 0.9707 & 0.9732 & 0.9620 & 0.9717 & 0.9885 & 0.9910 & 0.9878 & 0.9506 \\
\hline t-stat. & -1.0400 & -0.7525 & -0.7095 & -1.3295 & -0.9490 & -0.3474 & -0.2242 & -0.3141 & -0.9182 \\
\hline C.V. & -2.2813 & -2.4099 & -2.5779 & -2.6083 & -2.5919 & -2.6426 & -2.6033 & -2.6255 & -2.5969 \\
\hline \multicolumn{10}{|l|}{$\mathrm{H}-\mathrm{L}$} \\
\hline QKS test & 1.3295 & & & & & & & & \\
\hline \multicolumn{10}{|c|}{ SOUTH_KOREA } \\
\hline$\alpha(\tau)$ & 0.9331 & 0.9772 & 1.0000 & 0.9995 & 0.9816 & 0.9875 & 1.0286 & 1.0360 & 1.0922 \\
\hline t-stat. & -0.2738 & -0.3781 & 0.0000 & -0.0197 & -0.9710 & -0.6194 & 1.1941 & 1.2901 & 0.8997 \\
\hline C.V. & -2.7253 & -2.6947 & -2.5161 & -2.5130 & -2.4604 & -2.6048 & -2.5335 & -2.4288 & -2.1851 \\
\hline \multicolumn{10}{|l|}{$\mathrm{H}-\mathrm{L}$} \\
\hline QKS test & 1.2901 & & & & & & & & \\
\hline \multicolumn{10}{|c|}{ MEXICO } \\
\hline$\alpha(\tau)$ & 0.9898 & 1.0015 & 0.9643 & 0.9726 & 0.9731 & 0.9037 & 0.8872 & 0.8441 & 0.8591 \\
\hline t-stat. & -0.1594 & 0.0284 & -0.6940 & -0.4489 & -0.4009 & -1.4347 & -1.5218 & -1.7091 & -1.4547 \\
\hline C.V. & -2.2180 & -2.7351 & -2.5577 & -2.7430 & -2.6009 & -2.6567 & -2.7449 & -2.7213 & -2.5415 \\
\hline \multicolumn{10}{|l|}{$\mathrm{H}-\mathrm{L}$} \\
\hline QKS test & 1.7091 & & & & & & & & \\
\hline \multicolumn{10}{|c|}{ SAUDI_ARABIA } \\
\hline$\alpha(\tau)$ & 0.8712 & 0.9552 & 0.9560 & 0.9846 & 0.9829 & 1.0125 & 1.0384 & 1.0397 & 0.9764 \\
\hline t-stat. & -2.0418 & -0.6838 & -0.6044 & -0.2394 & -0.3664 & 0.2466 & 0.6934 & 0.6999 & -2.6294 \\
\hline C.V. & -2.5288 & -2.2667 & -2.2937 & -2.5542 & -2.4994 & -2.4805 & -2.4279 & -2.2780 & -2.4223 \\
\hline \multicolumn{10}{|l|}{$\mathrm{H}-\mathrm{L}$} \\
\hline QKS test & 2.6294 & & & & & & & & \\
\hline
\end{tabular}




\begin{tabular}{|c|c|c|c|c|c|c|c|c|c|}
\hline Quantile & 0.10 & 0.20 & 0.30 & 0.40 & 0.50 & 0.60 & 0.70 & 0.80 & 0.90 \\
\hline \multicolumn{10}{|c|}{ SOUTH_AFRICA } \\
\hline$\alpha(\tau)$ & 0.7803 & 0.9309 & 0.8916 & 0.9023 & 0.9337 & 0.9289 & 0.8472 & 0.8798 & 0.6815 \\
\hline t-stat. & -1.3936 & -0.6037 & -1.1685 & -1.3767 & -0.7444 & -0.7468 & -1.4588 & -0.9266 & -2.7507 \\
\hline C.V. & -2.1200 & -2.5897 & -2.7156 & -2.7464 & -2.8060 & -2.7231 & $\mathrm{NaN}$ & $\mathrm{NaN}$ & -2.7586 \\
\hline \multicolumn{10}{|l|}{$\mathrm{H}-\mathrm{L}$} \\
\hline QKS test & 2.7507 & & & & & & & & \\
\hline \multicolumn{10}{|c|}{ TURKEY } \\
\hline$\alpha(\tau)$ & 1.0408 & 1.0388 & 0.9808 & 0.9763 & 0.9691 & 0.9603 & 0.9344 & 0.9062 & 0.8784 \\
\hline t-stat. & 1.2807 & 0.8864 & -0.4382 & -0.5604 & -0.6891 & -0.9426 & -1.5285 & -1.5654 & -1.8216 \\
\hline C.V. & -2.4837 & -2.7391 & -2.7457 & -2.7343 & -2.7228 & -2.8144 & -2.8035 & -2.8399 & -2.5367 \\
\hline \multicolumn{10}{|l|}{$\mathrm{H}-\mathrm{L}$} \\
\hline QKS test & 1.8216 & & & & & & & & \\
\hline \multicolumn{10}{|c|}{ UK } \\
\hline$\alpha(\tau)$ & 0.9834 & 0.9864 & 0.9986 & 0.9782 & 0.9843 & 0.9764 & 0.9804 & 0.9866 & 1.0023 \\
\hline t-stat. & -0.4296 & -0.3415 & -0.0355 & -1.1599 & -0.8655 & -1.3627 & -1.2137 & -0.5043 & 0.0903 \\
\hline C.V. & -2.1200 & -2.3295 & -2.2066 & -2.2472 & -2.2946 & -2.3265 & -2.1200 & -2.1200 & -2.1200 \\
\hline \multicolumn{10}{|l|}{$\mathrm{H}-\mathrm{L}$} \\
\hline QKS test & 1.3627 & & & & & & & & \\
\hline \multicolumn{10}{|c|}{ US } \\
\hline$\alpha(\tau)$ & 0.9784 & 0.9055 & 0.8457 & 0.8638 & 0.9496 & 0.9871 & 1.0225 & 1.0335 & 1.0567 \\
\hline t-stat. & -0.2525 & -0.9370 & -1.4994 & -1.4186 & -0.6946 & -0.2292 & 0.4410 & 0.5951 & 0.6280 \\
\hline C.V. & -2.5226 & $\mathrm{NaN}$ & -2.7407 & -2.8131 & -2.7758 & -2.6886 & -2.5764 & -2.5812 & -2.5641 \\
\hline \multicolumn{10}{|l|}{$\mathrm{H}-\mathrm{L}$} \\
\hline QKS test & 1.4994 & & & & & & & & \\
\hline
\end{tabular}

Notes: The table shows point estimates, $t$-statistics and critical values for the $5 \%$ significance level. If the t-statistic is numerically smaller than the critical value then we reject the null hypothesis of $\alpha(\tau)=1$ at the $5 \%$ level. QKS is the quantile Kolmogorov-Smirnov test. 2.7837 is $5 \%$ critical value for QKS based on 10000 bootstrapping simulations. $H-L=\ln (0.5) / \ln (\alpha(\tau))$ 
Table 3: Quantile Unit Root Test Results

\begin{tabular}{|c|c|c|c|c|c|c|c|c|c|}
\hline Quantile & 0.10 & 0.20 & 0.30 & 0.40 & 0.50 & 0.60 & 0.70 & 0.80 & 0.90 \\
\hline \multicolumn{10}{|c|}{ ARGENTINA } \\
\hline$\alpha(\tau)$ & 0.9661 & 0.9732 & 0.9771 & 0.9704 & 0.9215 & 0.9252 & 0.9309 & 0.9297 & 0.9389 \\
\hline t-stat. & $\begin{array}{c}- \\
0.8274\end{array}$ & $\begin{array}{c}- \\
0.5614\end{array}$ & $\begin{array}{c}- \\
0.4711 \\
\end{array}$ & $\begin{array}{c}- \\
0.6029\end{array}$ & $\begin{array}{c}- \\
1.8852 \\
\end{array}$ & $\begin{array}{c}- \\
1.8834 \\
\end{array}$ & $\begin{array}{c}- \\
2.1075\end{array}$ & $\begin{array}{c}- \\
1.7649\end{array}$ & $\begin{array}{c}- \\
1.9117\end{array}$ \\
\hline C.V. & $\begin{array}{c}- \\
2.1764\end{array}$ & $\begin{array}{c}- \\
2.5266\end{array}$ & $\begin{array}{c}- \\
2.5097\end{array}$ & $\begin{array}{c}- \\
2.5269\end{array}$ & $\begin{array}{c}- \\
2.7231\end{array}$ & $\begin{array}{c}- \\
2.7050\end{array}$ & $\begin{array}{c}- \\
2.7015\end{array}$ & $\begin{array}{c}- \\
2.5657\end{array}$ & $\begin{array}{c}- \\
2.1566\end{array}$ \\
\hline $\mathrm{H}-\mathrm{L}$ & & & & & & & & & \\
\hline QKS test & 2.1075 & & & & & & & & \\
\hline \multicolumn{10}{|c|}{ AUSTRALIA } \\
\hline$\alpha(\tau)$ & 0.8668 & 0.9433 & 0.9627 & 0.9989 & 1.0079 & 0.9976 & 1.0014 & 1.0313 & 0.9942 \\
\hline t-stat. & $\begin{array}{c}- \\
2.8689\end{array}$ & $\begin{array}{c}- \\
1.1761\end{array}$ & $\begin{array}{c}- \\
1.0092\end{array}$ & $\begin{array}{c}- \\
0.0363\end{array}$ & 0.2307 & $\begin{array}{c}- \\
0.0592\end{array}$ & 0.0350 & 0.7690 & $\begin{array}{c}- \\
0.1863\end{array}$ \\
\hline C.V. & $\begin{array}{c}- \\
2.1223\end{array}$ & $\begin{array}{c}- \\
2.3384\end{array}$ & $\begin{array}{c}- \\
2.3177\end{array}$ & $\begin{array}{c}- \\
2.3684\end{array}$ & $\begin{array}{c}- \\
2.5553\end{array}$ & $\begin{array}{c}- \\
2.4369\end{array}$ & $\begin{array}{c}- \\
2.4363\end{array}$ & $\begin{array}{c}- \\
2.4058\end{array}$ & $\begin{array}{c}- \\
2.5978\end{array}$ \\
\hline $\mathrm{H}-\mathrm{L}$ & 4.8490 & & & & & & & & \\
\hline QKS test & 2.8689 & & & & & & & & \\
\hline \multicolumn{10}{|c|}{ BRAZIL } \\
\hline$\alpha(\tau)$ & 0.9135 & 0.9727 & 0.9644 & 0.9605 & 0.9395 & 0.9328 & 0.9264 & 0.8879 & 0.8933 \\
\hline t-stat. & 2.2238 & $\begin{array}{c}- \\
0.9993\end{array}$ & $\begin{array}{c}- \\
0.8917\end{array}$ & $\begin{array}{c}- \\
0.9768\end{array}$ & $\begin{array}{c}- \\
1.3118\end{array}$ & $\begin{array}{c}- \\
1.5389\end{array}$ & $\begin{array}{c}- \\
1.5555\end{array}$ & $\begin{array}{c}- \\
3.2906\end{array}$ & 2.5070 \\
\hline C.V. & $\mathrm{NaN}$ & $\begin{array}{c}- \\
2.5711\end{array}$ & $\begin{array}{c}- \\
2.5295\end{array}$ & $\begin{array}{c}- \\
2.5509\end{array}$ & $\begin{array}{c}- \\
2.7354\end{array}$ & $\begin{array}{c}- \\
2.6301\end{array}$ & $\begin{array}{c}- \\
2.4354\end{array}$ & $\begin{array}{c}- \\
2.2589\end{array}$ & $\begin{array}{c}- \\
2.1200\end{array}$ \\
\hline $\mathrm{H}-\mathrm{L}$ & & & & & & & & 5.8299 & 6.1431 \\
\hline QKS test & 3.2906 & & & & & & & & \\
\hline \multicolumn{10}{|c|}{ CANADA } \\
\hline$\alpha(\tau)$ & 0.8521 & 0.8247 & 0.7136 & 0.7488 & 0.7320 & 0.8771 & 0.7665 & 0.8969 & 0.9712 \\
\hline t-stat. & 1.1848 & $\begin{array}{c}- \\
1.9871\end{array}$ & $\begin{array}{c}- \\
2.9618\end{array}$ & $\begin{array}{c}- \\
2.3415\end{array}$ & $\begin{array}{c}- \\
2.2833\end{array}$ & $\begin{array}{c}- \\
0.9545\end{array}$ & $\begin{array}{c}- \\
1.9287\end{array}$ & $\begin{array}{c}- \\
1.0372\end{array}$ & 0.2423 \\
\hline C.V. & $\begin{array}{c}- \\
2.1200\end{array}$ & $\begin{array}{c}- \\
2.1666\end{array}$ & $\begin{array}{c}- \\
2.5194\end{array}$ & $\begin{array}{c}- \\
2.5998\end{array}$ & $\begin{array}{c}- \\
2.5390\end{array}$ & $\begin{array}{c}- \\
2.5201\end{array}$ & $\begin{array}{c}- \\
2.4995\end{array}$ & $\begin{array}{c}- \\
2.5494\end{array}$ & $\begin{array}{c}- \\
2.2732\end{array}$ \\
\hline $\mathrm{H}-\mathrm{L}$ & & & 2.0542 & & & & & & \\
\hline QKS test & 2.9618 & & & & & & & & \\
\hline \multicolumn{10}{|c|}{ CHINA } \\
\hline$\alpha(\tau)$ & 0.9495 & 0.9510 & 0.9613 & 0.9620 & 0.9517 & 0.9755 & 0.9853 & 0.9675 & 0.9810 \\
\hline t-stat. & 1.3411 & $\begin{array}{c}- \\
1.4842\end{array}$ & $\begin{array}{c}- \\
0.8324\end{array}$ & $\begin{array}{c}- \\
0.8558\end{array}$ & $\begin{array}{c}- \\
1.0129\end{array}$ & $\begin{array}{c}- \\
0.4543\end{array}$ & $\begin{array}{c}- \\
0.3050\end{array}$ & $\begin{array}{c}- \\
0.7372\end{array}$ & 0.4116 \\
\hline C.V. & $\begin{array}{c}- \\
2.1623\end{array}$ & $\begin{array}{c}- \\
2.4754\end{array}$ & $\begin{array}{c}- \\
2.6013\end{array}$ & $\begin{array}{c}- \\
2.6007\end{array}$ & $\begin{array}{c}- \\
2.5590\end{array}$ & $\begin{array}{c}- \\
2.6002\end{array}$ & $\begin{array}{c}- \\
2.5378\end{array}$ & $\begin{array}{c}- \\
2.6220\end{array}$ & $\begin{array}{c}- \\
2.1619\end{array}$ \\
\hline H-L & & & & & & & & & \\
\hline QKS test & 1.4842 & & & & & & & & \\
\hline \multicolumn{10}{|c|}{ FRANCE } \\
\hline$\alpha(\tau)$ & 0.9046 & 1.0049 & 0.9972 & 1.0270 & 1.0124 & 1.0205 & 1.0344 & 1.0302 & 1.0562 \\
\hline t-stat. & $\begin{array}{c}- \\
2.5287\end{array}$ & 0.1359 & $\begin{array}{c}- \\
0.0660\end{array}$ & 0.8226 & 0.4270 & 0.6685 & 0.9295 & 0.5788 & 1.1610 \\
\hline C.V. & - & - & - & - & - & - & - & - & - \\
\hline
\end{tabular}




\begin{tabular}{|c|c|c|c|c|c|c|c|c|c|}
\hline & 2.1200 & 2.5197 & 2.5745 & 2.6188 & 2.6965 & 2.5316 & 2.4366 & 2.4882 & 2.4015 \\
\hline \multicolumn{10}{|l|}{$\mathrm{H}-\mathrm{L}$} \\
\hline QKS test & 2.5287 & & & & & & & & \\
\hline \multicolumn{10}{|c|}{ GERMANY } \\
\hline$\alpha(\tau)$ & 0.8928 & 0.9900 & 1.0357 & 1.0210 & 1.0377 & 1.0649 & 1.0484 & 1.1044 & 1.0205 \\
\hline t-stat. & $\begin{array}{c}- \\
3.0939\end{array}$ & $\begin{array}{c}- \\
0.2199\end{array}$ & 0.7643 & 0.4152 & 0.6766 & 1.1679 & 0.7858 & 1.7156 & 0.8584 \\
\hline C.V. & $\begin{array}{c}- \\
2.1200\end{array}$ & $\begin{array}{c}- \\
2.4799 \\
\end{array}$ & $\begin{array}{c}- \\
2.4019\end{array}$ & $\begin{array}{c}- \\
2.3369\end{array}$ & $\begin{array}{c}- \\
2.7263 \\
\end{array}$ & $\begin{array}{c}- \\
2.3269\end{array}$ & $\begin{array}{c}- \\
2.6242\end{array}$ & $\begin{array}{c}- \\
2.5759\end{array}$ & $\begin{array}{c}- \\
2.6298 \\
\end{array}$ \\
\hline $\mathrm{H}-\mathrm{L}$ & 6.1128 & & & & & & & & \\
\hline QKS test & 3.0939 & & & & & & & & \\
\hline Quantile & 0.10 & 0.20 & 0.30 & 0.40 & 0.50 & 0.60 & 0.70 & 0.80 & 0.90 \\
\hline \multicolumn{10}{|c|}{ INDIA } \\
\hline$\alpha(\tau)$ & 0.9170 & 0.9180 & 0.9701 & 0.9627 & 0.9395 & 0.9409 & 0.9102 & 0.9048 & 0.9084 \\
\hline t-stat. & 2.1674 & $\begin{array}{c}- \\
3.8928 \\
\end{array}$ & $\begin{array}{c}- \\
0.9980 \\
\end{array}$ & $\begin{array}{c}- \\
1.1679 \\
\end{array}$ & $\begin{array}{c}- \\
1.3144 \\
\end{array}$ & $\begin{array}{c}- \\
1.3208 \\
\end{array}$ & $\begin{array}{c}- \\
2.2636 \\
\end{array}$ & $\begin{array}{c}- \\
2.8945 \\
\end{array}$ & 2.1813 \\
\hline C.V. & $\begin{array}{c}- \\
2.1200\end{array}$ & $\begin{array}{c}- \\
2.3407\end{array}$ & $\begin{array}{c}- \\
2.5786 \\
\end{array}$ & $\begin{array}{c}- \\
2.6704\end{array}$ & $\begin{array}{c}- \\
2.6836 \\
\end{array}$ & $\mathrm{NaN}$ & $\begin{array}{c}- \\
2.6345\end{array}$ & $\begin{array}{c}- \\
2.4115 \\
\end{array}$ & $\begin{array}{c}- \\
2.1200 \\
\end{array}$ \\
\hline $\mathrm{H}-\mathrm{L}$ & & 8.1015 & & & & & & 6.9286 & \\
\hline QKS test & 3.8928 & & & & & & & & \\
\hline \multicolumn{10}{|c|}{ INDONESIA } \\
\hline$\alpha(\tau)$ & 1.0219 & 0.9768 & 0.9614 & 0.9729 & 0.9546 & 0.9688 & 0.9461 & 0.9239 & 0.9256 \\
\hline t-stat. & 0.6591 & $\begin{array}{c}- \\
0.6750\end{array}$ & $\begin{array}{c}- \\
1.2855 \\
\end{array}$ & $\begin{array}{c}- \\
0.8549\end{array}$ & $\begin{array}{c}- \\
1.5424\end{array}$ & $\begin{array}{c}- \\
0.9747\end{array}$ & $\begin{array}{c}- \\
1.5801 \\
\end{array}$ & $\begin{array}{c}- \\
2.0276 \\
\end{array}$ & $\begin{array}{c}- \\
1.0474 \\
\end{array}$ \\
\hline C.V. & $\begin{array}{c}- \\
2.3523\end{array}$ & $\begin{array}{c}- \\
2.4066\end{array}$ & $\begin{array}{c}- \\
2.3981\end{array}$ & $\begin{array}{c}- \\
2.5919\end{array}$ & $\begin{array}{c}- \\
2.6943\end{array}$ & $\begin{array}{c}- \\
2.7439\end{array}$ & $\begin{array}{c}- \\
2.6585\end{array}$ & $\begin{array}{c}- \\
2.6246\end{array}$ & $\begin{array}{c}- \\
2.4679\end{array}$ \\
\hline $\mathrm{H}-\mathrm{L}$ & & & & & & & & & \\
\hline QKS test & 2.0276 & & & & & & & & \\
\hline \multicolumn{10}{|c|}{ ITALY } \\
\hline$\alpha(\tau)$ & 0.9220 & 0.9130 & 0.8997 & 0.9225 & 0.9497 & 0.9587 & 0.9744 & 0.9825 & 1.0142 \\
\hline t-stat. & $\begin{array}{c}- \\
1.6623\end{array}$ & $\begin{array}{c}- \\
1.8234\end{array}$ & $\begin{array}{c}- \\
2.2130\end{array}$ & $\begin{array}{c}- \\
1.8912\end{array}$ & $\begin{array}{c}- \\
1.2524\end{array}$ & $\begin{array}{c}- \\
0.9026\end{array}$ & $\begin{array}{c}- \\
0.4971\end{array}$ & $\begin{array}{c}- \\
0.2785\end{array}$ & 0.2290 \\
\hline C.V. & $\begin{array}{c}- \\
2.3510 \\
\end{array}$ & $\begin{array}{c}- \\
2.1949 \\
\end{array}$ & $\begin{array}{c}- \\
2.4582 \\
\end{array}$ & $\begin{array}{c}- \\
2.2848 \\
\end{array}$ & $\begin{array}{c}- \\
2.3489 \\
\end{array}$ & $\begin{array}{c}- \\
2.5287 \\
\end{array}$ & $\begin{array}{c}- \\
2.6111 \\
\end{array}$ & $\begin{array}{c}- \\
2.5746 \\
\end{array}$ & $\begin{array}{c}- \\
2.2989\end{array}$ \\
\hline $\mathrm{H}-\mathrm{L}$ & & & & & & & & & \\
\hline QKS test & 2.2130 & & & & & & & & \\
\hline \multicolumn{10}{|c|}{ JAPAN } \\
\hline$\alpha(\tau)$ & 0.9623 & 0.9707 & 0.9732 & 0.9620 & 0.9717 & 0.9885 & 0.9910 & 0.9878 & 0.9506 \\
\hline t-stat. & $\begin{array}{c}- \\
1.0400 \\
\end{array}$ & $\begin{array}{c}- \\
0.7525 \\
\end{array}$ & $\begin{array}{c}- \\
0.7095 \\
\end{array}$ & $\begin{array}{c}- \\
1.3295 \\
\end{array}$ & $\begin{array}{c}- \\
0.9490 \\
\end{array}$ & $\begin{array}{c}- \\
0.3474 \\
\end{array}$ & $\begin{array}{c}- \\
0.2242 \\
\end{array}$ & $\begin{array}{c}- \\
0.3141 \\
\end{array}$ & $\begin{array}{c}- \\
0.9182 \\
\end{array}$ \\
\hline C.V. & $\begin{array}{c}- \\
2.2813 \\
\end{array}$ & $\begin{array}{c}- \\
2.4099 \\
\end{array}$ & $\begin{array}{c}- \\
2.5779 \\
\end{array}$ & $\begin{array}{c}- \\
2.6083 \\
\end{array}$ & $\begin{array}{c}- \\
2.5919 \\
\end{array}$ & $\begin{array}{c}- \\
2.6426 \\
\end{array}$ & $\begin{array}{c}- \\
2.6033 \\
\end{array}$ & $\begin{array}{c}- \\
2.6255 \\
\end{array}$ & $\begin{array}{c}- \\
2.5969 \\
\end{array}$ \\
\hline $\mathrm{H}-\mathrm{L}$ & & & & & & & & & \\
\hline QKS test & 1.3295 & & & & & & & & \\
\hline
\end{tabular}




\begin{tabular}{|c|c|c|c|c|c|c|c|c|c|}
\hline$\alpha(\tau)$ & 0.9331 & 0.9772 & 1.0000 & 0.9995 & 0.9816 & 0.9875 & 1.0286 & 1.0360 & 1.0922 \\
\hline t-stat. & $\begin{array}{c}- \\
0.2738\end{array}$ & $\begin{array}{c}- \\
0.3781\end{array}$ & 0.0000 & $\begin{array}{c}- \\
0.0197\end{array}$ & $\begin{array}{c}- \\
0.9710\end{array}$ & $\begin{array}{c}- \\
0.6194\end{array}$ & 1.1941 & 1.2901 & 0.8997 \\
\hline C.V. & $\begin{array}{c}- \\
2.7253\end{array}$ & $\begin{array}{c}- \\
2.6947\end{array}$ & $\begin{array}{c}- \\
2.5161\end{array}$ & $\begin{array}{c}- \\
2.5130\end{array}$ & $\begin{array}{c}- \\
2.4604\end{array}$ & $\begin{array}{c}- \\
2.6048\end{array}$ & $\begin{array}{c}- \\
2.5335\end{array}$ & $\begin{array}{c}- \\
2.4288\end{array}$ & $\begin{array}{c}- \\
2.1851\end{array}$ \\
\hline H-L & & & & & & & & & \\
\hline QKS test & 1.2901 & & & & & & & & \\
\hline \multicolumn{10}{|c|}{ MEXICO } \\
\hline$\alpha(\tau)$ & 0.9898 & 1.0015 & 0.9643 & 0.9726 & 0.9731 & 0.9037 & 0.8872 & 0.8441 & 0.8591 \\
\hline t-stat. & $\begin{array}{c}- \\
0.1594\end{array}$ & 0.0284 & $\begin{array}{c}- \\
0.6940\end{array}$ & $\begin{array}{c}- \\
0.4489\end{array}$ & $\begin{array}{c}- \\
0.4009\end{array}$ & $\begin{array}{c}- \\
1.4347\end{array}$ & $\begin{array}{c}- \\
1.5218\end{array}$ & $\begin{array}{c}- \\
1.7091\end{array}$ & $\begin{array}{c}- \\
1.4547\end{array}$ \\
\hline C.V. & $\begin{array}{c}- \\
2.2180\end{array}$ & $\begin{array}{c}- \\
2.7351\end{array}$ & $\begin{array}{c}- \\
2.5577\end{array}$ & $\begin{array}{c}- \\
2.7430\end{array}$ & $\begin{array}{c}- \\
2.6009\end{array}$ & $\begin{array}{c}- \\
2.6567\end{array}$ & $\begin{array}{c}- \\
2.7449\end{array}$ & $\begin{array}{c}- \\
2.7213\end{array}$ & $\begin{array}{c}- \\
2.5415\end{array}$ \\
\hline $\mathrm{H}-\mathrm{L}$ & & & & & & & & & \\
\hline QKS test & 1.7091 & & & & & & & & \\
\hline \multicolumn{10}{|c|}{ SAUDI_ARABIA } \\
\hline$\alpha(\tau)$ & 0.8712 & 0.9552 & 0.9560 & 0.9846 & 0.9829 & 1.0125 & 1.0384 & 1.0397 & 0.9764 \\
\hline t-stat. & $\begin{array}{c}- \\
2.0418\end{array}$ & $\begin{array}{c}- \\
0.6838\end{array}$ & $\begin{array}{c}- \\
0.6044\end{array}$ & $\begin{array}{c}- \\
0.2394\end{array}$ & $\begin{array}{c}- \\
0.3664\end{array}$ & 0.2466 & 0.6934 & 0.6999 & $\begin{array}{c}- \\
2.6294\end{array}$ \\
\hline C.V. & $\begin{array}{c}- \\
2.5288\end{array}$ & $\begin{array}{c}- \\
2.2667\end{array}$ & $\begin{array}{c}- \\
2.2937\end{array}$ & $\begin{array}{c}- \\
2.5542\end{array}$ & $\begin{array}{c}- \\
2.4994\end{array}$ & $\begin{array}{c}- \\
2.4805\end{array}$ & $\begin{array}{c}- \\
2.4279\end{array}$ & $\begin{array}{c}- \\
2.2780\end{array}$ & $\begin{array}{c}- \\
2.4223 \\
\end{array}$ \\
\hline \multicolumn{10}{|l|}{$\mathrm{H}-\mathrm{L}$} \\
\hline QKS test & 2.6294 & & & & & & & & \\
\hline
\end{tabular}

\begin{tabular}{|c|c|c|c|c|c|c|c|c|c|}
\hline Quantile & 0.10 & 0.20 & 0.30 & 0.40 & 0.50 & 0.60 & 0.70 & 0.80 & 0.90 \\
\hline \multicolumn{10}{|c|}{ SOUTH_AFRICA } \\
\hline$\alpha(\tau)$ & 0.7803 & 0.9309 & 0.8916 & 0.9023 & 0.9337 & 0.9289 & 0.8472 & 0.8798 & 0.6815 \\
\hline t-stat. & $\begin{array}{c}- \\
1.3936\end{array}$ & $\begin{array}{c}- \\
0.6037\end{array}$ & $\begin{array}{c}- \\
1.1685\end{array}$ & $\begin{array}{c}- \\
1.3767\end{array}$ & $\begin{array}{c}- \\
0.7444\end{array}$ & $\begin{array}{c}- \\
0.7468\end{array}$ & $\begin{array}{c}- \\
1.4588\end{array}$ & $\begin{array}{c}- \\
0.9266\end{array}$ & $\begin{array}{c}- \\
2.7507\end{array}$ \\
\hline C.V. & $\begin{array}{c}- \\
2.1200\end{array}$ & $\begin{array}{c}- \\
2.5897 \\
\end{array}$ & $\begin{array}{c}- \\
2.7156\end{array}$ & $\begin{array}{c}- \\
2.7464\end{array}$ & $\begin{array}{c}- \\
2.8060\end{array}$ & $\begin{array}{c}- \\
2.7231 \\
\end{array}$ & $\mathrm{NaN}$ & $\mathrm{NaN}$ & $\begin{array}{c}- \\
2.7586 \\
\end{array}$ \\
\hline \multicolumn{10}{|l|}{$\mathrm{H}-\mathrm{L}$} \\
\hline QKS test & 2.7507 & & & & & & & & \\
\hline \multicolumn{10}{|c|}{ TURKEY } \\
\hline$\alpha(\tau)$ & 1.0408 & 1.0388 & 0.9808 & 0.9763 & 0.9691 & 0.9603 & 0.9344 & 0.9062 & 0.8784 \\
\hline t-stat. & 1.2807 & 0.8864 & $\begin{array}{c}- \\
0.4382\end{array}$ & $\begin{array}{c}- \\
0.5604\end{array}$ & $\begin{array}{c}- \\
0.6891\end{array}$ & $\begin{array}{c}- \\
0.9426\end{array}$ & $\begin{array}{c}- \\
1.5285\end{array}$ & $\begin{array}{c}- \\
1.5654\end{array}$ & $\begin{array}{c}- \\
1.8216\end{array}$ \\
\hline C.V. & $\begin{array}{c}- \\
2.4837\end{array}$ & $\begin{array}{c}- \\
2.7391\end{array}$ & $\begin{array}{c}- \\
2.7457\end{array}$ & $\begin{array}{c}- \\
2.7343\end{array}$ & $\begin{array}{c}- \\
2.7228\end{array}$ & $\begin{array}{c}- \\
2.8144\end{array}$ & $\begin{array}{c}- \\
2.8035\end{array}$ & $\begin{array}{c}- \\
2.8399\end{array}$ & $\begin{array}{l}- \\
2.5367\end{array}$ \\
\hline $\mathrm{H}-\mathrm{L}$ & & & & & & & & & \\
\hline QKS test & 1.8216 & & & & & & & & \\
\hline \multicolumn{10}{|c|}{ UK } \\
\hline$\alpha(\tau)$ & 0.9834 & 0.9864 & 0.9986 & 0.9782 & 0.9843 & 0.9764 & 0.9804 & 0.9866 & 1.0023 \\
\hline t-stat. & $\begin{array}{c}- \\
0.4296\end{array}$ & $\begin{array}{c}- \\
0.3415\end{array}$ & $\begin{array}{c}- \\
0.0355\end{array}$ & $\begin{array}{c}- \\
1.1599\end{array}$ & $\begin{array}{c}- \\
0.8655\end{array}$ & $\begin{array}{c}- \\
1.3627\end{array}$ & $\begin{array}{c}- \\
1.2137\end{array}$ & $\begin{array}{c}- \\
0.5043\end{array}$ & 0.0903 \\
\hline
\end{tabular}




\begin{tabular}{|l|c|c|c|c|c|c|c|c|c|}
\hline C.V. & - & - & - & - & - & - & - & - & - \\
& 2.1200 & 2.3295 & 2.2066 & 2.2472 & 2.2946 & 2.3265 & 2.1200 & 2.1200 & 2.1200 \\
\hline H-L & & & & & & & & & \\
\hline QKS test & 1.3627 & & & \multicolumn{7}{|c|}{} & & & & & \\
\hline \multicolumn{8}{|l|}{ US } \\
\hline$\alpha(\tau)$ & 0.9784 & 0.9055 & 0.8457 & 0.8638 & 0.9496 & 0.9871 & 1.0225 & 1.0335 & 1.0567 \\
\hline t-stat. & - & - & - & - & - & - & 0.4410 & 0.5951 & 0.6280 \\
& 0.2525 & 0.9370 & 1.4994 & 1.4186 & 0.6946 & 0.2292 & & & \\
\hline C.V. & - & NaN & - & - & - & - & - & - & - \\
& 2.5226 & & 2.7407 & 2.8131 & 2.7758 & 2.6886 & 2.5764 & 2.5812 & 2.5641 \\
\hline H-L & & & & & & & & & \\
\hline QKS test & 1.4994 & & & & & & & & \\
\hline
\end{tabular}

Notes: The table shows point estimates, t-statistics and critical values for the $5 \%$ significance level.

If the t-statistic is numerically smaller than the critical value then we reject the null hypothesis of $\alpha(\tau)=1$ at the $5 \%$ level. QKS is the quantile Kolmogorov-Smirnov test. 2.7837 is $5 \%$ critical value for QKS based on 10000 bootstrapping simulations. $H-L=\ln (0.5) / \ln (\alpha(\tau))$ 
This means a slower speed of adjustment after a shock in the high quantile level.

On the other hand, we find that the persistent point estimate is slightly lower at the higher tail quantiles for Australia, Brazil, India, Indonesia, Japan, Mexico, South Africa and Turkey and the estimated half-life of a shock. Also, it decreases from lower quantile to higher quantile, meaning a slower speed of adjustment after a shock in the lower quantile level. These results indicate that shocks to relative per capita $\mathrm{CO}_{2}$ emissions are nonlinear and asymmetric. These results are not shown in previous studies. The asymmetric adjusting speed reveals time-varying persistence perceptions and high market sensitivity in these G18 countries.

It is worth noting that our results here are not consistent with those of Wang and Zhang (2014), Wang et al., (2014), Hao et al., (2015), and Zhao et al. (2015). All these four studies point that relative per capita $\mathrm{CO}_{2}$ emissions in China based on either provincial data or all sectors are converged from 1990s to 2012. Our study is consistent with those of Aldy (2006a) and Ye et al. (2017) that they found strong evidence of divergence for the whole sample and some evidence of convergence for the OECD countries sample.

For only five out of 18 countries, our study finds stronger evidence in support relative per capita $\mathrm{CO}_{2}$ emissions convergence. The $\mathrm{CO}_{2}$ convergence means that the markets can equilibrium actively and the policymakers can stick to current policies, and this means that the policymakers can reduce carbon dioxide emission rigidly according to the difference of emissions amount in Australia, Brazil, Canada, Germany and India. Regarding the divergence economies such as Argentina, China, France, 
Indonesia, Italy, Japan, Mexico, Saudi Arabia, South Africa, South Korea, Turkey, the UK and the US can take some measures to reduce emission amounts to release the burden of massive usage of fossil energy and to adjust economic instruments. Policy proposals in these countries must reexamine the existing carbon-reducing policies and propose some reasonable suggestions to obtain sustainable development (Sun et al., 2016; Ye et al., 2017).

The non-convergence in these 13 countries also shows that the global energy system is not mature enough to incorporate internationally implemented, common policies and strategies. As noted by McKibbin and Stegman (2005) that one of the major reasons for considering the existence of emissions convergence a crucial topic for researchers and policymakers is the ability for projections. Environmental policies will be better formed if convergence (even conditional) did exist. Based our empirical results, the projection for these 13 economies' $\mathrm{CO} 2$ emissions (i.e., Argentina, China, France, Indonesia, Italy, Japan, Mexico, Saudi Arabia, South Africa, South Korea, Turkey, the UK and the US) cannot be estimated with precision. As a result, the policies should also be country-specific and will depend on the socio-economic characteristics of each country (El-Montasser et al., 2015; Ye et al., 2017). For the other five countries such as Australia, Brazil, Canada, Germany and India where relative $\mathrm{CO}_{2}$ emissions converge and this implies the existing policies in these five countries are rational. The policymakers of these economies can continue with their current carbon-reduction policies. 


\section{Conclusions}

By making use of the Quantile Unit Root test, this paper investigates whether $\mathrm{CO}_{2}$ emissions converge in G18 countries using relative per capita $\mathrm{CO}_{2}$ emissions data over 1950-2013. While traditional unit root test results indicate relative per capita $\mathrm{CO}_{2}$ emissions, do not converge among G18 countries. Results from Quantile Unit root test point out that strong $\mathrm{CO}_{2}$ emission converge in Australia, Brazil, Canada, Germany and India (5 of the 18 countries). Although we do not find strong relative per capita $\mathrm{CO}_{2}$ emission converge in the other 13 countries (i.e., Argentina, China, France, Indonesia, Italy, Japan, Mexico, Saudi Arabia, South Africa, South Korea, Turkey, the UK and the US), the relative per capita $\mathrm{CO}_{2}$ emissions did converge in certain quantiles for some of these 13 countries. Our study has important policy implications for the governments of these G18 countries to implement the effective energy policy to reduce the $\mathrm{CO}_{2}$ emissions. 


\section{References}

Aldy, J.E (2006b) Per capita carbon dioxide emissions: convergence or divergence? Environmental Resource Economics, 33:533-555

Aldy, J.E. (2006a) Divergence in State-Level Per Capita Carbon Dioxide Emissions, Discussion Paper, RFF DP 06-07.

Barassi, M. R., Cole, M. A. and Elliott, R. J. R. (2008) Stochastic divergence or convergence of per capita carbon dioxide emissions: re-examining the evidence, Environmental and Resource Economics, 40, 121-37.

Barassi, M. R., Cole, M. A. and Elliott, R. J. R. (2011) The stochastic convergence of CO2 emissions: a long memory approach, Environmental and Resource Economics, 49, 367-85.

Camarero, M, Mendoza, Y and Ordoñez, J (2011) Re-examining emissions. Is assessing convergence meaning less? Department of Applied Economics II Universidad de Valencia Working Papers 1104:1-38.

Chang, C. P and Lee, C. C. (2008) Are per capita carbon dioxide emissions converging among industrialized countries? New time series evidence with structural breaks, Environment and Development Economics, 13, 4, 497-515.

Dickey, D. A. and Fuller, W. A. (1980) Likelihood ratio statistics for autoregressive time series with a unit root, Econometrica, 49: 1057-1072.

EI-Monstasser, G., Inglesi-Lotz, R., Gupta, R. (2015) Convergence of Greenhouse Gas Emissions among G7 Countrie, Applied Economics, 47, 60, 6543-6552.

Evans, P. (1998) Using panel data to evaluate growth theories, International Economic Review, 39:295-306

Hao, Y. Zhang, Q. Zhong, M. and Li, B (2015) Is there convergence in per capita SO2 emissions in China? An empirical study using city-level panel data, Journal of Cleaner Production, 108, 944-954.

Huang, B and Meng, Lina (2013) Convergence of per capita carbon dioxide emissions in Urban China: a spatio-temporal perspective, Applied Geography, 40, 21-29.

Jobert, T., Karanfil, F. and Tykhonenko, A. (2010) Convergence of per capita carbon dioxide emissions in the EU: Legend or reality? Energy Economics, 32, 1364-1373.

Koenker, R. and Xiao, Z. (2004). Unit Root Quantile Autoregression Inference. Journal of the American Statistical Association, 99, 775-787.

Kwiatkowski, D., Phillips, P., Schmidt, P., and Shin, Y (1992), "Testing the Null Hypothesis of Stationarity Against the Alternative of a Unit Root: How Sure Are We That Economic Time Series Have a Unit Root?” Journal of Econometrics, 54, 159-178.

Lee, C.C, and Chang, C.P. (2009) Stochastic convergence of per capita carbon dioxide emissions and multiple structural breaks in OECD countries. Economic Modelling, 26(6):1375-1381

Li, X. and Lin, B. (2013) Global convergence in per capita CO2 emissions, enewable and Sustainable Energy Reviews, 24, 357-63. 
Li, X-L., Tang, D.P and Chang, TY (2014) CO2 emissions converge in the 50 U.S. states Sequential panel selection method, Economic Modelling, 40, 320-333.

McKibbin,W. J. and Stegman, A. (2005) Convergence and per capita carbon emissions, Brooking Discussion papers in International Economics No 167.

Ordas Criado, C. and Grether, J.M., (2011) Convergence in per capita CO2 emissions: a robust distributional approach. Resource Energy Economics. 33, 637-665

Panopoulou, E. and Pantelidis, T. (2009) Club convergence in carbon dioxide emissions, Environmental and Resource Economics, 44, 47-70

Phillips, P. C. B., and P. Perron (1988) Testing for a Unit Root in Time Series Regression." Biometrika 75, 335-346.

Romero-Ávila, D. (2008) Convergence in carbon dioxide emissions among industrialised countries revisited, Energy Economics, 30, 2265-2282.

Strazicich, MC, and List, J. A (2003) Are CO2 emissions level converging among industrial countries? Environmental and Resource Economics, 24:263-271

Sun, J., Chi-Wei Su and Gui-lan Shao (2016) Is carbon dioxide emission convergence in ten largest economies? International Journal of Green Energy, 13,5,454-461.

Wang, J. and Zhang, K. (2014) Convergence of carbon dioxide emissions in different sectors in China, Energy, 65, 605-611.

Wang, Y. Zhang, P. Huang, D. and Cai, C. (2014) Convergence behavior of carbon dioxide emissions in China, Economic Modelling, 43, 75-80.

Westerlund, J. and Basher, S.A. (2007) Testing for convergence in carbon dioxide emissions using a century of panel data. Environmental and Resource Economics, 40, 1, 109-120.

Yavuz, Nilgun Cil and Yilanci, Veli. (2013) Convergence in Per Capita Carbon Dioxide Emissions among G7 Countries: A TAR Panel Unit Root Approach. Environmental and Resource Economics, February 2013, 54:2, pp. 283-91.

Zhao, X. Burnett, J. W. and Lacombe, D. J (2015) Province-level convergence of China's carbon dioxide emissions, Applied Energy, 150, 286-295. 